\title{
Lift induced by slip inhomogeneities in lubricated contacts
}

\author{
Aidan Rinehart $\odot,{ }^{1}$ Uğis Lācis $\odot,{ }^{1}$ Thomas Salez, ${ }^{2,3}$ and Shervin Bagheri ${ }^{1, *}$ \\ ${ }^{1}$ Department of Engineering Mechanics, KTH Royal Institute of Technology, Stockholm SE-10044, Sweden \\ ${ }^{2}$ University of Bordeaux, CNRS, LOMA, UMR 5798, F-33405, Talence, France \\ ${ }^{3}$ Global Station for Soft Matter, Gi-CoRE, Hokkaido University, Sapporo, Hokkaido 060-0808, Japan
}

(Received 3 December 2019; revised 27 April 2020; accepted 20 July 2020;

published 11 August 2020)

\begin{abstract}
Lubrication forces depend to a high degree on elasticity, texture, charge, chemistry, and temperature of the interacting surfaces. Therefore, by appropriately designing surface properties, we may tailor lubrication forces to reduce friction, adhesion, and wear between sliding surfaces and control repulsion, assembly, and collision of interacting particles. Here, we show that variations of slippage on one of the contacting surfaces induce a lift force. We demonstrate the consequences of this force on the mobility of a cylinder traveling near a wall and show the emergence of particle oscillation and migration that would not otherwise occur in the Stokes flow regime. Our study has implications for understanding how inhomogeneous biological interfaces interact with their environment; we also propose a method of patterning surfaces for controlling the motion of nearby particles.
\end{abstract}

DOI: 10.1103/PhysRevFluids.5.082001

In many physical processes, the flow of small particles such as cells, colloids, bubbles, and fibers occurs near soft, porous, and rough walls. The induced lubrication forces [1] on these particles depend on the elasticity, texture, and chemistry of the nearby wall. These forces may dominate over both bulk (e.g., Stokes drag) and surface (e.g., van der Waals and electrostatic) forces and therefore determine single-particle motion and collective behavior.

The simplest configuration to characterize hydrodynamic particle-wall forces is that of an infinitely long circular cylinder traveling parallel to a rigid flat wall. At low Reynolds numbers, a rigid cylinder will experience zero wall-normal (lift) force and therefore move at a constant distance from the wall [2]. This is due to the time-reversal symmetry of the Stokes equations. However, if one of the interacting surfaces is soft, the moving particle will be repelled from the wall [3] as a result of the broken symmetry of the fluid pressure in the thin gap [4-9]. This elastohydrodynamic lift mechanism increases the gap thickness and reduces wear and friction between the sliding surfaces [10-12]. It underlies exotic particle trajectories such as oscillations, Magnus-like effect, stick-slip motion, and spinning $[13,14]$. Soft lubrication also underpins surface rheology [15-17] that is used to characterize the viscoelasticity of complex surfaces.

Besides softness, another ubiquitous feature of surfaces in biology and technology is surface inhomogeneities. For example, the surface of a Janus particle is divided into two halves with different chemistries (hydrophobic-hydrophilic) or texture (rough-smooth). This provides the particle

\footnotetext{
*Corresponding author: shervin@mech.kth.se
}

Published by the American Physical Society under the terms of the Creative Commons Attribution 4.0 International license. Further distribution of this work must maintain attribution to the author(s) and the published article's title, journal citation, and DOI. Funded by Bibsam. 

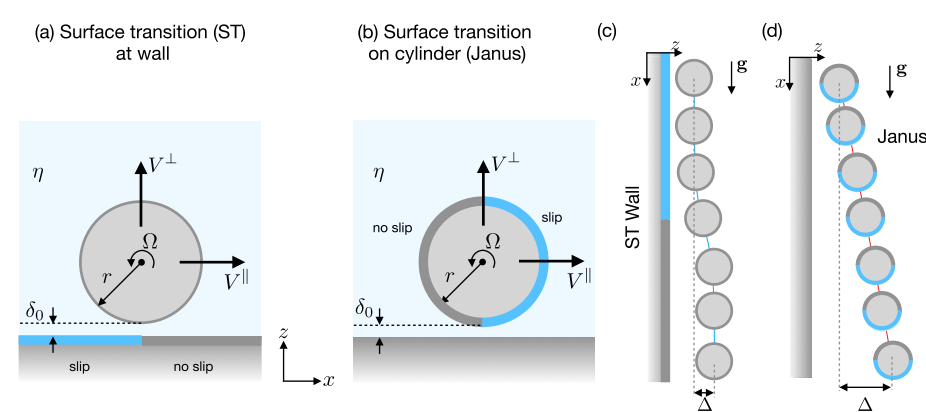

(e)

FIG. 1. Two lubrication models. (a) 2D solid cylinder moving near a flat wall with a transition from slip (blue) to no slip (gray). (b) 2D cylinder half-coated with a slip region and moving near a wall. [(c), (d)] Sketches of cylinder trajectories for the two systems with $\delta_{0}=0.05 r, \ell=1.79 \delta_{0}$ and cylinder density $\rho_{\mathrm{c}}=8.77 \rho$. The particle experiences a small rotation that is not visually observable. (e) Gap thickness vs the transverse displacement of the cylinder. Inset shows the trajectory over a larger spatial extent.

with unique capabilities including self-assembly into complex structures [18] and self-propulsion [19]. More generally, interfaces in living tissues (cell walls, blood vessels, cartilage, epithelia) vary in chemical and mechanical composition due to inhomogeneous distribution of cells and proteins. In technological applications, inhomogeneous surfaces arise as a consequence of manufacturing imperfections and wear, but also from surface patterning to control liquid transport [20] or heat transfer [21]. Despite this ubiquity, there has been no investigation of the full set of lubrication forces arising from particle-wall interaction when the properties of one of the contacting surfaces vary.

In this Rapid Communication, we study lubrication forces when slippage [22] properties change along the contacting surfaces. We consider a model of spatially varying slip length $\ell$ at either the surface of a flat wall or the surface of a cylinder [Figs. 1(a) and 1(b)]. Here, $\ell$ is defined as an effective property of the interface at some coarse-grained level. The slip length can be considered as a mesoscopic model emerging from small-scale features such as surface charges [23], wall roughness [24], superhydrophobicity [25], liquid infusion [26], and temperature or solute concentration gradients $[27,28]$.

Using analytical and numerical treatments, we demonstrate that surface inhomogeneities give rise to particle trajectories such as oscillations, migration, and propulsion. Underlying these phenomena is a normal lift force that arises from spatial variations in surface slippage. To illustrate this, consider the two configurations in Figs. 1(c) and 1(d). Both cases involve a cylinder of radius $r$ located a distance $\delta_{0}$ from a flat wall and immersed in a fluid with viscosity $\eta$ and density $\rho$. We assume small Reynolds number, $\operatorname{Re}=\rho V r / \eta \ll 1$, where $V$ is the characteristic velocity of the cylinder.

Figure 1(e) (blue) shows the trajectory of the cylinder falling freely under gravity next to a wall that has a single slip transition. The trajectory is obtained from numerical simulations of Stokes equations coupled to Newton's equation of motion for the cylinder (see the Supplemental Material [29]) with density ratio between cylinder density and fluid density $\rho_{c} / \rho=8.77$. For this test, gravity force is included in the Newton's equation of motion. As the cylinder passes the transition line, it migrates away from the wall a distance $\Delta$, which is comparable to $\delta_{0}$. In contrast, a wall with homogeneous slippage produces zero lift force (and consequently no wall-normal motion) on a cylinder [30]. Therefore, the lift arises here from the sudden change in slip length at the wall. By carrying out an expansion in the dimensionless slip length $L=\ell / \delta_{0}$, we will show that the lift force per unit length of the cylinder scales as $F_{z} \sim \eta V^{\|} \varepsilon^{-1} L$ at the transition line and that $\Delta \sim \ell$, where $\varepsilon=$ $\delta_{0} / r \ll 1$. Importantly, this lift force can be comparable in magnitude to other lubrication forces. For instance, for a red blood cell traveling near glycocalyx [31], variations of the slip length as small as a few nanometers induce a lift force comparable to the elastohydrodynamic one $(\approx 0.1 \mathrm{pN})$ caused by glycocalyx deformation [32]. 
(a) Freely falling cylinder near a patterned wall

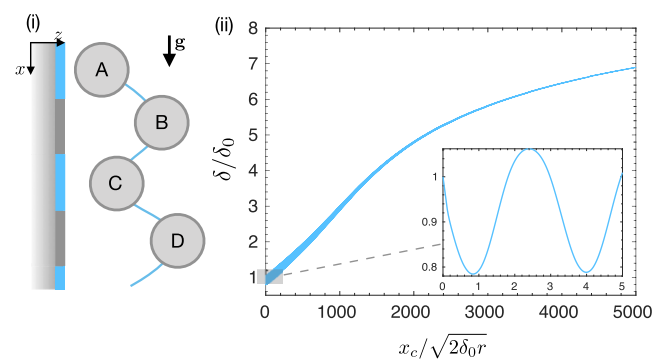

(b) Imposed rotation on neutrally buoyant cylinder above a surface

(i)

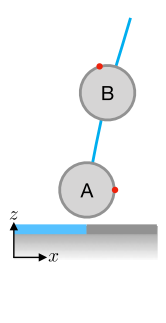

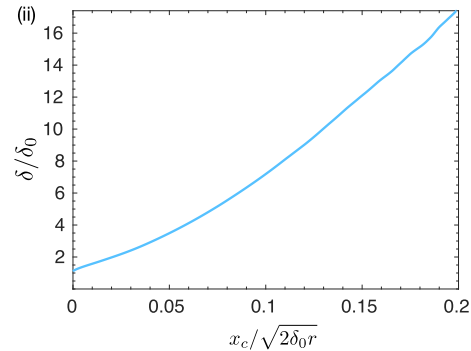

(c) Imposed rotation on neutrally buoyant Janus particle

(i)

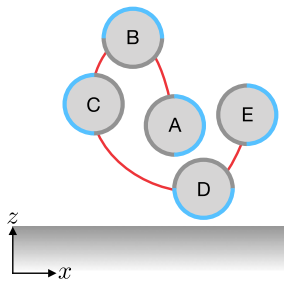

(ii)

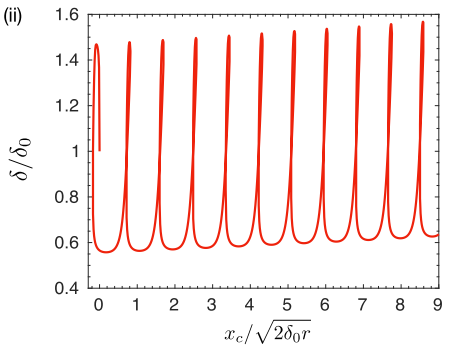

FIG. 2. Cylinder trajectories induced by variation of slippage. (a) Cylinder with density ratio $\rho_{c} / \rho=8.77$ falling near a wall that alternates between slip (blue) and no slip (gray). (b) Neutrally buoyant cylinder $\left(\rho_{c} / \rho=\right.$ 1) rotating above a slip-to-no-slip transition. (c) Neutrally buoyant Janus cylinder $\left(\rho_{c} / \rho=1\right)$ rotating next to a wall. Right column (ii) shows the gap thickness as a function of the transverse displacement. For all configurations, $\delta_{0} / r=0.05$ and $L=1.79$.

Figure 1(e) (red) shows the trajectory of a Janus cylinder with density $\rho_{c}=8.77 \rho$ falling near a flat wall [Fig. 1(d)] under the influence of gravity. We observe a persistent normal drift along the trajectory, since the transition from slip to no slip is now located on the traveling cylinder itself, thus constantly inducing a wall-normal force. Scaling estimates, that will be obtained below, indicate that $\Delta \sim x_{c} \ell / l_{\mathrm{c}}$, where $l_{\mathrm{c}}=\sqrt{2 \delta_{0} r}$ is the lubrication contact length and $x_{c}$ is the transverse displacement. As $\Delta$ becomes larger than $r$, one expects a saturation of normal migration, since lubrication forces become negligible in the bulk. Nevertheless, as shown in the inset of Fig. 1(e), the effect holds for $\delta$ substantially larger than $r$.

The coupling of rigid-body motion to slippage inhomogeneities can result in unexpected particle dynamics (Fig. 2). In the following, we will study in detail these motions using lubrication theory and scaling laws. At low Reynolds numbers, the force per unit length, $\mathbf{F}=\left(F_{x}, F_{z}\right)$, and torque per unit length, $T$, on the cylinder are linearly related to the velocity $\mathbf{V}=\left(V^{\|}, V^{\perp}\right)$ and the angular speed $\Omega$ of the cylinder. This is expressed by the symmetric resistance matrix [33],

$$
\left[\begin{array}{c}
F_{x} \\
F_{z} \\
T
\end{array}\right]=-\eta\left[\begin{array}{ccc}
f_{x}^{\|} & -f_{x}^{\perp} & r f_{x}^{\omega} \\
-f_{z}^{\|} & f_{z}^{\perp} & -r f_{z}^{\omega} \\
r t^{\|} & -r t^{\perp} & r^{2} t^{\omega}
\end{array}\right]\left[\begin{array}{c}
V^{\|} \\
V^{\perp} \\
\Omega
\end{array}\right]
$$

where $f_{x}^{\perp}=f_{z}^{\|}, t^{\|}=f_{x}^{\omega}$, and $t^{\perp}=f_{z}^{\omega}$. Assuming a small gap, $\varepsilon=\delta_{0} / r \ll 1$, we explain below the procedure to determine the elements of the resistance matrix for the cylinder translating parallel to the wall with a slip-to-no-slip transition [Fig. 3(a)(ii)].

We nondimensionalize the variables as

$$
x=l_{\mathrm{c}} X, \quad z=\delta_{0} Z, \quad h=\delta_{0} H, \quad u=V^{\|} U, \quad w=\frac{\varepsilon^{1 / 2} V^{\|}}{\sqrt{2}} W, \quad p=\frac{l_{\mathrm{c}} \eta V^{\|}}{\delta_{0}^{2}} P,
$$




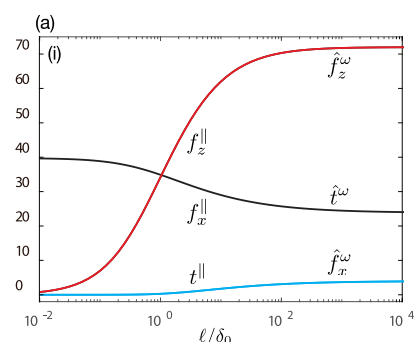

(ii)

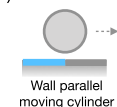

(iii)

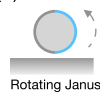

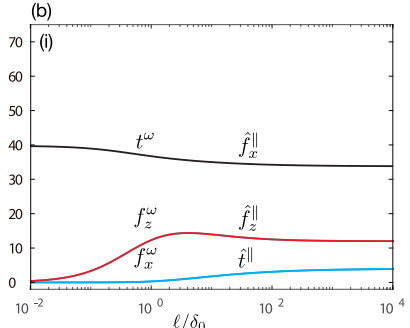

(iii)

(ii)

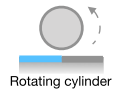

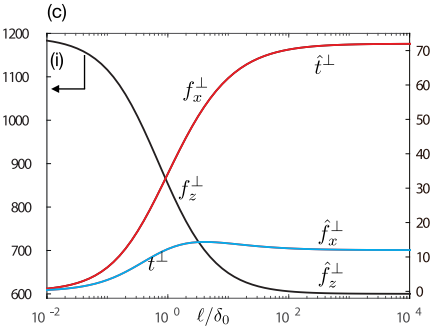

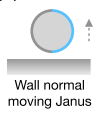

(ii)

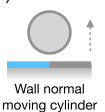

(iii)

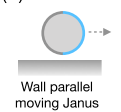

FIG. 3. Analytical resistance coefficients for both model systems $(\varepsilon=0.05)$. In the inset (i), the coefficients with no^refer to configuration (ii) and the ones with^refer to (iii).

where $u, w$ are, respectively, the transverse and normal components of the fluid velocity and $p$ is the fluid excess pressure with respect to the atmospheric one. The cylinder surface is approximated as $h(x)=\delta_{0}+x^{2} /(2 r)$. Inserting the dimensionless variables into the continuity and Stokes equations and neglecting $\mathcal{O}(\varepsilon)$ terms, we obtain

$$
\partial_{X} P=\partial_{Z Z} U, \quad \partial_{Z} P=0, \quad \partial_{X} U+\partial_{Z} W=0 .
$$

In the laboratory frame of reference, the boundary conditions are

$$
\left.W\right|_{Z=0, H}=0,\left.\quad U\right|_{Z=H}=1,\left.\quad U\right|_{Z=0}=\left.L \partial_{Z} U\right|_{Z=0} S .
$$

The horizontal velocity at the wall $\left.U\right|_{Z=0}$ (3) accounts for slippage and is modeled through a Navier boundary condition. Moreover, $S$ equals one for $X<0$ and zero for $X>0$, where $X=0$ is the location of the transition from slip to no slip. The solution of Eqs. (2) and (3) is a combination of Couette and Poiseuille flows (see the Supplemental Material [29]). From that solution, the fluid stress is projected onto the cylinder surface to obtain

$$
f_{x}^{\|}=\sqrt{2} \varepsilon^{-1 / 2} \int_{-\infty}^{\infty}\left(2 X P+\partial_{Z} U\right) d X, \quad f_{z}^{\|}=2 \varepsilon^{-1} \int_{-\infty}^{\infty} P d X, \quad t^{\|}=\sqrt{2} \varepsilon^{-1 / 2} \int_{-\infty}^{\infty} \partial_{Z} U d X .
$$

Figure 3(a)(i) shows the elements [Eq. (4)] of the resistance matrix as a function of dimensionless slip length. When $\ell / \delta_{0} \rightarrow 0$, only the drag coefficient $\left(f_{x}^{\|}\right)$is nonzero, in agreement with the results for no-slip surfaces [2]. For larger $\ell / \delta_{0}$, we note the emergence of nonzero elements related to lift force $\left(f_{z}^{\|}\right)$[as observed in Fig. 1(e)] and torque $t^{\|}$.

Figure 3(b)(i) shows the elements (denoted by the^symbol) of a Janus cylinder that translates parallel to a wall. We again observe the emergence of off-diagonal terms of the resistance matrix for $\ell \approx \delta_{0}$; in particular, the lift force $\left(\hat{f}_{z}^{\|}\right)$causing the constant normal migration shown in Fig. 1(e). The complete set of elements for both model systems is reported in Fig. 3. Note that due to the Lorentz reciprocal theorem [33] there is a symmetry between the two model systems.

To understand in detail the lift-induced mechanism of slip-to-no-slip transitions, we study the gap pressure for three different slip lengths. Figure 4(a) shows the pressure distribution for a moving cylinder over an inhomogeneous wall [trajectory in Fig. 1(c)]. The no-slip solution maintains an antisymmetric distribution (red). The introduction of a finite slip length breaks this symmetry (blue and black), as the gap pressure necessary to accelerate the flow through the gap (with varying thickness) is increased over the slippery section $(X<0)$. Figure 4(b) shows the gap pressure of the wall-parallel moving Janus particle [trajectory in Fig. 1(d)]. Here, the induced lubrication pressure needs to accommodate both the varying gap thickness and the varying fluid shear in the gap, which results in a pressure peak over the slippery section of the Janus cylinder. Note that due to the 
(a) Wall parallel moving cylinder

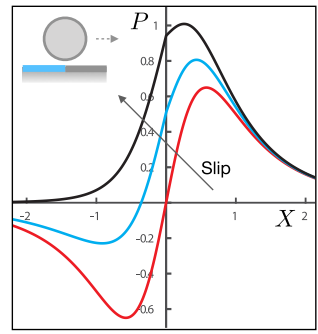

(b) Wall parallel moving Janus

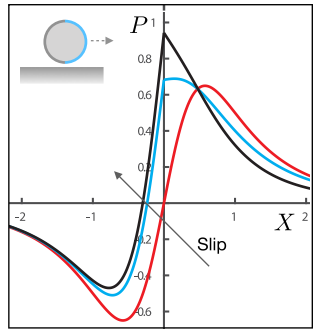

(c) Rotating Janus

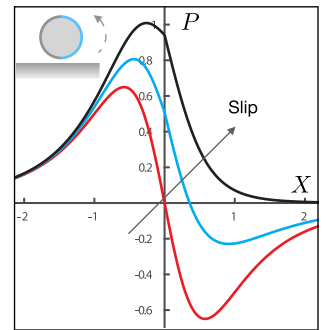

(d) Rotating cylinder

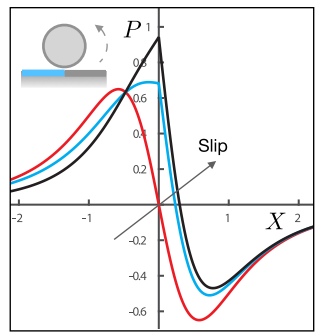

FIG. 4. Pressure distributions in four configurations and for three slip lengths, $L=0$ (red), $L=1$ (blue), and $L=\infty$ (black).

symmetry between the two configurations the distributions in Figs. 4(a) and 4(b) are the mirror of those in Figs. 4(c) and 4(d), respectively.

We now explain the trajectories in Fig. 2 using the components of the resistance matrix. In Fig. 2(a), a slip-to-no-slip transition pushes the cylinder away from the wall [Fig. 1(c)], whereas a no-slip-to-slip transition produces a negative $F_{z}$, pulling the cylinder toward the wall. The long-term net migration away from the wall [Fig. 2(a)(ii)] is partially due to the fact that the push force is slightly larger than the pull force for each period due to different gap thicknesses during the pull and push events (see the Supplemental Material [29]).

The second example involves a rotating neutrally buoyant cylinder [Fig. 2(b)]. When the cylinder is released above a slippage transition on the wall, we observe a migration in $x$ and $z$ directions [Fig. 2(b)(ii)]. The resistance coefficients in Figs. 3(b) and 3(c) explain this behavior. The imposed rotation produces a lift force $\left(F_{z} \sim \eta r \Omega f_{z}^{\omega}>0\right)$ and a negative transverse thrust $\left(F_{x} \sim-\eta r \Omega f_{x}^{\omega}<0\right)$. However, as the cylinder migrates away from the wall, we have $V^{\perp}>0$, which leads to a positive transverse thrust $\left(F_{x} \sim \eta V^{\perp} f_{x}^{\perp}>0\right)$. The $V^{\perp}$-generated thrust dominates over the $\Omega$-generated thrust, such that the cylinder moves in the positive $x$ direction.

The final example involves a rotating neutrally buoyant Janus cylinder [Fig. 2(c)]. The cylinder undergoes a spiralling motion that results in positive transverse propulsion and positive wall-normal migration. We explain the motion in stages A-E depicted in Fig. 2(c). In A, the cylinder migrates upward since $\hat{f}_{z}^{\omega}>0$. It simultaneously migrates to the left since, due to the short exposure, $V^{\perp}$ is relatively small and we have $\left|V^{\perp} \hat{f}_{x}^{\perp}\right|<\left|r \Omega \hat{f}_{x}^{\omega}\right|$. In B, the cylinder has rotated such that no slippage remains in the gap. Therefore, a lift force is no longer generated and the wall-normal drag force $\hat{f}_{z}^{\perp}$ hinders further upward migration. Stages $\mathrm{C}$ and $\mathrm{D}$ are the mirror of $\mathrm{A}$ and $\mathrm{B}$, respectively. Consequently, we observe a migration in the negative $z$ and positive $x$ directions. As the cylinder reaches stage $\mathrm{E}$, it has experienced a net translation in the positive $x$ direction and a small net migration away from the wall from its initial position A. This is due to the difference in the magnitude of fluid stresses in the gap when slip (B) and no-slip (D) surfaces face the wall.

Finally, we turn to scaling analysis to estimate the normal displacement induced by the lift force. We first consider cylinder motion over inhomogeneous wall. We focus on the instant where a cylinder is located above the transition from slip to no slip on the wall [Fig. 1(a)]. We assume no rotation, constant transverse, and wall-normal velocities $V^{\|}$and $V^{\perp}$, and negligible inertial effects (see the Supplemental Material [29]). A wall-normal force balance yields $\eta f_{z}^{\|} V^{\|}=\eta f_{z}^{\perp} V^{\perp}$, corresponding to the magnitude of the lift and drag components, respectively. By carrying out an expansion in $L \ll 1$ of $f_{z}^{\|}$and $f_{z}^{\perp}$, we can approximate the two coefficients using leading-order terms as

$$
f_{z}^{\|} \simeq 4 \ell r / \delta_{0}^{2} \quad \text { and } \quad f_{z}^{\perp} \simeq-3 \sqrt{2} \pi\left(\delta_{0} / r\right)^{-3 / 2}
$$


By assuming that the lift force approximately acts over $l_{\mathrm{c}}$, we have

$$
V^{\|}=l_{\mathrm{c}} / \tau \text { and } \quad V^{\perp}=\Delta / \tau
$$

where $\tau$ is the time it takes to traverse $l_{\mathrm{c}}$, and $\Delta$ is the displacement from the wall. Inserting these estimates in the wall-normal force balance yields $\Delta \sim \ell$. This is in agreement with numerical results shown Fig. 1(c), where we observe $\Delta \approx \delta_{0}$ for $\ell \approx \delta_{0}$. For a Janus cylinder [Fig. 1(b)], there is no fixed time over which the displacement occurs, because the transition point travels along with the cylinder. Therefore, we expect a displacement $\approx \ell$ for each $l_{\mathrm{c}}$ traversed. This yields

$$
\Delta \sim \ell x_{\mathrm{c}} / l_{\mathrm{c}}
$$

where $x_{\mathrm{c}}$ is the distance traversed along the wall. Next, we consider the scaling law for neutrally buoyant cylinder migration due to imposed rotation. We start with the slip-to-no-slip transition on the wall [Fig. 2(b)(i)]. Let us define the net migration over one revolution of the cylinder. We estimate the cylinder angular velocity as $\Omega \sim \pi / \tau_{2}$ and wall-normal velocity as $V^{\perp} \sim \Delta / \tau_{2}$, where $\tau_{2}$ is the time it takes for the cylinder to do one revolution. We make use of the wall-normal force balance again, which yields $f_{z}^{\omega} \eta r \Omega=f_{z}^{\perp} \eta V^{\perp}$. The force coefficients are again approximated with a leading-order term from Taylor series expansion for $L \ll 1$, i.e.,

$$
f_{z}^{\omega} \simeq 2 \ell r / \delta_{0}^{2} \text { and } f_{z}^{\perp} \simeq-3 \sqrt{2} \pi\left(\delta_{0} / r\right)^{-3 / 2} .
$$

Inserting all the expressions for force coefficients and cylinder velocities in the force balance yields

$$
\Delta \sim \ell \sqrt{\frac{r}{\delta_{0}}} .
$$

Interestingly, the leading-order scaling estimate for rotating cylinder provides a displacement which decays as the gap thickness is increased. This is a physically intuitive result, since the lubrication force from the wall is expected to decay as the gap thickness increases. This qualitatively explains the net migration away from the wall, observed for patterned inhomogeneous wall [Fig. 2(a)], although the scaling law for single slip-to-no-slip transition at the leading order does not contain gap thickness dependence. We would like to note that it is possible to include a higher order effect also in single slip-to-no-slip transition to capture the gap thickness effect (see the Supplemental Material [29]). However, we have found that for the translation above the inhomogeneous wall the wall-normal displacement dependence on the gap thickness is much weaker compared to the rotation above the slip-to-no-slip transition. Finally, we consider the rotating Janus particle located above a homogeneous wall. As the Janus particle rotates, the interaction between the surface inhomogeneity and the solid wall will vary over time. To obtain a scaling law, we simplify this process by dividing it up in two steps. In first, the orientation of the Janus particle is such that there is a positive wall-normal force and particle moves away from the wall. In the second step, the orientation of the Janus particle is turned by angle $\pi$ (or is mirrored) compared to the first step, and consequently there is a negative wall-normal force and particle moves toward the wall. Both of these steps we model using the instant where the slip-to-no-slip transition of the Janus particle is located closest to the wall. We estimate the cylinder angular velocity as $\Omega \sim \pi / \tau_{3}$ and wall-normal velocity as $V^{\perp} \sim \Delta / \tau_{3}$, where $\tau_{3}$ is the time it takes the Janus particle to turn by angle $\pi$. We employ the wall-normal force balance, which yields $\hat{f}_{z}^{\omega} \eta r \Omega=\hat{f}_{z}^{\perp} \eta V^{\perp}$. Taylor series expansion (see the Supplemental Material [29]) for the coefficients of the Janus particle gives

$$
\hat{f}_{z}^{\omega} \simeq 4 \ell r / \delta_{0}^{2} \text { and } \hat{f}_{z}^{\perp} \simeq-3 \sqrt{2} \pi\left(\delta_{0} / r\right)^{-3 / 2} .
$$

Inserting all the expressions in the force balance yields the same displacement estimate as for rotating cylinder above the inhomogeneous wall, Eq. (9),

$$
\Delta_{1} \sim \ell \sqrt{\frac{r}{\delta_{0}}}
$$


TABLE I. Scaling laws for lift force $\left(F_{z}\right)$ and wall-normal displacement $(\Delta)$ for ST wall or Janus particle. The imposed motion is either wall parallel velocity or rotation. For the latter, the displacement shown is for one revolution.

\begin{tabular}{lccc}
\hline \hline & Motion & Lift force & Displacement \\
\hline ST wall & $V^{\|}$ & $F_{z} \sim \eta V^{\|} \varepsilon^{-1} L$ & $\Delta \sim \ell$ \\
Janus & $V^{\|}$ & $F_{z} \sim \eta V^{\|} \varepsilon^{-1} L$ & $\Delta \sim \ell x_{\mathrm{c}} / l_{\mathrm{c}}$ \\
ST wall & $\Omega$ & $F_{z} \sim \eta r \Omega \varepsilon^{-1} L$ & $\Delta \sim \sqrt{r / \delta_{0}} \ell$ \\
Janus & $\Omega$ & $F_{z} \sim \eta r \Omega \varepsilon^{-1} L$ & $\Delta \sim \ell \sqrt{r / \delta_{0}}\left(1-\frac{1}{\sqrt{1+\ell r^{1 / 2} \delta_{0}^{-3 / 2}}}\right)$ \\
\hline \hline
\end{tabular}

but this time it is only for the first step or first half-revolution of the Janus particle. For the second step, the estimate can be obtained exactly the same way while remembering that the wall-normal force is negative due to change of surface inhomogeneity orientation, i.e., $\hat{f}_{z}^{\omega} \simeq-4 \ell r / \delta_{0}^{2}$. This gives us a negative displacement estimate for the second step

$$
\Delta_{2} \sim-\ell \sqrt{\frac{r}{\delta_{1}}},
$$

where starting gap thickness is different compared to the gap thickness at the beginning of the rotation $\delta_{0}$. The starting gap thickness for the second step can be obtained by adding the displacement of the first step to the initial gap thickness, which yields

$$
\delta_{1}=\delta_{0}+\Delta_{1}=\delta_{0}+\ell \sqrt{\frac{r}{\delta_{0}}} .
$$

Finally, the estimate for Janus particle displacement away from the wall over one revolution can be obtained by summing up displacements from both steps as

$$
\Delta=\Delta_{1}+\Delta_{2} \sim \ell \sqrt{\frac{r}{\delta_{0}}}\left(1-\frac{1}{\sqrt{1+\ell r^{1 / 2} \delta_{0}^{-3 / 2}}}\right) .
$$

Since slip length, radius, and gap thickness all have positive values, the term $\ell r^{1 / 2} \delta_{0}^{-3 / 2}$ is positive and the net displacement of the Janus particle over one revolution is positive, which agrees with our numerical results [Fig. 2(c)], in which we observe a net drift away from the wall. Table I summarizes the scaling estimates of wall normal displacement for both systems in Figs. 1(a) and 1(b).

To conclude, we have used numerical simulations, analytical results, and scaling arguments to describe how objects with spatially inhomogeneous slippage may encounter nontrivial emergent forces and torques. In the presence of small slippage inhomogeneities, our scaling estimate suggests that the lift force, $F_{z \text {,slip }}$, has to be considered alongside other lubrication forces. For instance, using atomic-force microscopy, the elastohydrodynamic force was reported [34] to be $F_{z \text {,EHD }} \sim \mathrm{nN}$. Considering the same experimental setup, but with a rigid substrate exhibiting an $\approx 1$-nm slip inhomogeneity, we obtain $F_{z \text {,slip }} \sim$ nN, i.e., the same order of magnitude as for the soft substrate [35].

Such slippage inhomogeneities may arise from small variations in surface preparation, localized defects, or cleaning processes, and this slippage has the potential to modify the observed wallnormal force. For example, in the experiments of a traveling Janus particle near a wall [36], variations of contact angle up to $10^{\circ}$ on the same substrate were reported. Finally, our numerical results for both gravity-driven and other type of configurations show how nontrivial particle trajectories can spontaneously emerge from slippage inhomogeneities. Note that the scaling estimates derived in this work are not specific to the particular type of driving force applied to the moving particle; it could be gravity, electromagnetic, or other type of force. The developments presented in this paper 
open up interesting opportunities for the design of interfaces to control and influence nearby particle motion, as well as to reduce friction and wear. It also provides a foundation to explore more realistic situations found in nature and especially in biology.

A.R., S.B., and U.L. were funded through the Knut and Alice Wallenberg Foundation (KAW 2016.0255), the Swedish Foundation for Strategic Research (SSF, FFL15:0001), and Swedish Research Council (VR-2014-5680, INTERFACE centre). We also thank Abdelhamid Maali and Juho Lintuvuori for valuable comments on the manuscript.

[1] O. Reynolds, IV. On the theory of lubrication and its application to Mr. Beauchamp Tower's experiments, including an experimental determination of the viscosity of olive oil, Philos. Trans. R. Soc. London 177, 157 (1886).

[2] D. Jeffery and Y. Onishi, The slow motion of a cylinder next to a plane wall, Q. J. Mech. Appl. Math. 34, 129 (1981).

[3] K. Sekimoto and L. Leibler, A mechanism for shear thickening of polymer-bearing surfaces: Elastohydrodynamic coupling, EPL 23, 113 (1993).

[4] I. Cantat and C. Misbah, Lift Force and Dynamical Unbinding of Adhering Vesicles Under Shear Flow, Phys. Rev. Lett. 83, 880 (1999).

[5] M. Abkarian, C. Lartigue, and A. Viallat, Tank Treading and Unbinding of Deformable Vesicles in Shear Flow: Determination of the Lift Force, Phys. Rev. Lett. 88, 068103 (2002).

[6] J. Beaucourt, T. Biben, and C. Misbah, Optimal lift force on vesicles near a compressible substrate, EPL 67, 676 (2004).

[7] J. M. Skotheim and L. Mahadevan, Soft Lubrication, Phys. Rev. Lett. 92, 245509 (2004).

[8] J. Urzay, S. G. Llewellyn Smith, and B. J. Glover, The elastohydrodynamic force on a sphere near a soft wall, Phys. Fluids 19, 103106 (2007).

[9] J. H. Snoeijer, J. Eggers, and C. H. Venner, Similarity theory of lubricated hertzian contacts, Phys. Fluids 25, 101705 (2013).

[10] B. Saintyves, T. Jules, T. Salez, and L. Mahadevan, Self-sustained lift and low friction via soft lubrication, Proc. Natl. Acad. Sci. U.S.A. 113, 5847 (2016).

[11] B. Rallabandi, N. Oppenheimer, M. Y. Ben Zion, and H. A. Stone, Membrane-induced hydroelastic migration of a particle surfing its own wave, Nat. Phys. 14, 1211 (2018).

[12] P. Vialar, P. Merzeau, S. Giasson, and C. Drummond, Compliant surfaces under shear: Elastohydrodynamic lift force, Langmuir 35, 15605 (2019).

[13] T. Salez and L. Mahadevan, Elastohydrodynamics of a sliding, spinning, and sedimenting cylinder near a soft wall, J. Fluid Mech. 779, 181 (2015).

[14] B. Rallabandi, B. Saintyves, T. Jules, T. Salez, C. Schönecker, L. Mahadevan, and H. A. Stone, Rotation of an immersed cylinder sliding near a thin elastic coating, Phys. Rev. Fluids 2, 074102 (2017).

[15] A. Steinberger, C. Cottin-Bizonne, P. Kleimann, and E. Charlaix, Nanoscale Flow on a Bubble Mattress: Effect of Surface Elasticity, Phys. Rev. Lett. 100, 134501 (2008).

[16] S. Leroy, A. Steinberger, C. Cottin-Bizonne, F. Restagno, L. Léger, and É. Charlaix, Hydrodynamic Interaction Between a Spherical Particle and an Elastic Surface: A Gentle Probe for Soft Thin Films, Phys. Rev. Lett. 108, 264501 (2012).

[17] Y. Wang, G. A. Pilkington, C. Dhong, and J. Frechette, Elastic deformation during dynamic force measurements in viscous fluids, Curr. Opin. Colloid Interface Sci. 27, 43 (2017).

[18] J. Yan, M. Bloom, S. Bae, E. Luijten, and S. Granick, Linking synchronization to self-assembly using magnetic janus colloids, Nature (London) 491, 578 (2012).

[19] R. Golestanian, T. B. Liverpool, and A. Ajdari, Propulsion of a Molecular Machine by Asymmetric Distribution of Reaction Products, Phys. Rev. Lett. 94, 220801 (2005). 
[20] J. S. Wexler, A. Grosskopf, M. Chow, Y. Fan, I. Jacobi, and H. A. Stone, Robust liquid-infused surfaces through patterned wettability, Soft Matter 11, 5023 (2015).

[21] D. Attinger, C. Frankiewicz, A. R. Betz, T. M. Schutzius, R. Ganguly, A. Das, C.-J. Kim, and C. M. Megaridis, Surface engineering for phase change heat transfer: A review, MRS Energy Sustain. 1, E4 (2014).

[22] E. Lauga, M. Brenner, and H. Stone, Microfluidics: The no-slip boundary condition, in Springer Handbook of Experimental Fluid Mechanics (Springer, Berlin, 2007), pp. 1219-1240.

[23] L. Bocquet and J.-L. Barrat, Flow boundary conditions from nano- to micro-scales, Soft Matter 3, 685 (2007).

[24] U. Lacis and S. Bagheri, A framework for computing effective boundary conditions at the interface between free fluid and a porous medium, J. Fluid Mech. 812, 866 (2017).

[25] J. P. Rothstein, Slip on superhydrophobic surfaces, Ann. Rev. Fluid Mech. 42, 89 (2010).

[26] T.-S. Wong, S. H. Kang, S. K. Y. Tang, E. J. Smythe, B. D. Hatton, A. Grinthal, and J. Aizenberg, Bioinspired self-repairing slippery surfaces with pressure-stable omniphobicity, Nature (London) 477, 443 (2011).

[27] J. L. Anderson, Colloid transport by interfacial forces, Ann. Rev. Fluid Mech. 21, 61 (1989).

[28] A. Ajdari and L. Bocquet, Giant Amplification of Interfacially Driven Transport by Hydrodynamic Slip: Diffusio-Osmosis and Beyond, Phys. Rev. Lett. 96, 186102 (2006).

[29] See Supplemental Material at http://link.aps.org/supplemental/10.1103/PhysRevFluids.5.082001 for details of resistance coefficient derivation, discussion on role of inertia, and description of numerical experiments, including reference to the numerical tool FreeFEM++ [37] .

[30] U. Kaynan and E. Yariv, Stokes resistance of a cylinder near a slippery wall, Phys. Rev. Fluids 2, 104103 (2017).

[31] Typical parameters are as follows: red blood cell radius $r=w 3 \mu \mathrm{m}$, cell-wall gap $\delta=0.5 \mu \mathrm{m}$, fluid viscosity $\eta=1.5 \mathrm{mPas}$, and speed $V^{\|}=0.286 \mathrm{mms}^{-1}$ estimated by multiplying the $100 \mathrm{~s}^{-1}$ shear rate and the cell radius [32].

[32] H. S. Davies, D. Débarre, N. El Amri, C. Verdier, R. P. Richter, and L. Bureau, Elastohydrodynamic Lift at a Soft Wall, Phys. Rev. Lett. 120, 198001 (2018).

[33] L. G. Leal, Advanced Transport Phenomena: Fluid Mechanics and Convective Transport Processes, Series: Cambridge Series in Chemical Engineering (Book 7) (Cambridge University Press, Cambridge, UK, 2007).

[34] Z. Zhang, V. Bertin, M. Arshad, E. Raphaël, T. Salez, and A. Maali, Direct Measurement of the Elastohydrodynamic Lift Force at the Nanoscale, Phys. Rev. Lett. 124, 054502 (2020).

[35] Experimental parameters from Ref. [34]: radius $r=60 \mu \mathrm{m}$, probe-wall gap $\delta=50 \mathrm{~nm}$, fluid viscosity $\eta=96 \mathrm{mPas}$, and speed $V^{\|}=0.57 \mathrm{mms}^{-1}$. Inserting these values in $F_{z}$ in Table I (first row) gives $F_{z, \text { slip }}=$ $F_{z} l_{c} \sim 1 \mathrm{nN}$. Here, $l_{c}=\sqrt{2 R \delta}$ is the relevant length scale for the lift force.

[36] S. Ketzetzi, J. de Graaf, R. P. Doherty, and D. J. Kraft, Slip Length Dependent Propulsion Speed of Catalytic Colloidal Swimmers Near Walls, Phys. Rev. Lett. 124, 048002 (2020).

[37] F. Hecht, New development in FreeFEM++, J. Numer. Math. 20, 251 (2012). 